\title{
EDITORIAL
}

\section{The little database that could: Intermountain Health Care and the uphill quest for prevention of term necrotizing enterocolitis}

Journal of Perinatology (2007) 27, 397-398; doi:10.1038/sj.jp.7211756

The cohort study by Lambert et al. ${ }^{1}$ in this edition of The Journal of Perinatology will stand the test of time as being one of those smart studies with a small cohort of interest (just 30 affected patients), that gave the field of neonatology some important answers. First of all, they asked a specific and targeted question. They asked what was unique about term infants who acquire necrotizing enterocolitis (NEC) from their counterparts with regard to clinical risk factors. It is an old question, but they asked it in a manner that was unambiguous. Once they identified those variables, they then turned the tables and identified all other infants who also had those risk factors and did not acquire NEC (400 from a total 5847 patients). These two cohorts were then compared for their feeding patterns; that is, who was fed breast milk exclusively, who got formula exclusively, who got both. There was a significant association with exclusive formula feeding and NEC and a comparable association between exclusive breast-feeding and the absence of NEC. In fact, every single NEC case had some exposure to a formula product.

This was the most important finding in the paper, and I'll come back to it, but there's more in this little juggernaut. The study is, to my knowledge and that of the authors, the first cohort study ever done on term NEC. They made the most of it and have dispelled a number of myths in the literature. The first is the myth that term NEC presents in the first 2 or 3 days of life. Their data showed that the peak time of presentation is between days 6 and 10. The second myth is that there is an anatomic vascular insufficiency in one of the arteries supplying the ascending colon. However, every single patient in this cohort had a pre-existing illness (either small for gestational age, congenital heart disease, polycythemia or depression at birth) that could be hypothesized to perturb mesenteric blood flow through hypoxic-ischemic physiology. The third is that the disease is predominantly a surgical disease. Less than a quarter of their cohort required surgery. Finally, the authors 'speculated that reduced mesenteric perfusion and feeding with artificial formula were factors predisposing them to develop NEC.' I think they're on firm footing with that one.

Gail Besner is a co-author on this paper and she's also on a key paper of Philip Nowicki, ${ }^{2}$ who has fathered the research on endothelin- 1 and its role in the pathogenesis of NEC. ${ }^{3,4}$ Dr Besner is a convenient link in the research chain to focus on (even though she is more well known as the discoverer of

heparin-binding epidermal growth factor), because endothelin-1 participates in the pathogenesis of all forms of NEC. However, endothelin-1 may be unique to the initiation of term NEC, which was uniformly associated with hypoxic-ischemic preconditions in the Intermountain Health Care cohort.

Interestingly enough, a very nice animal study demonstrating this relationship was recently published. ${ }^{5}$ The experiments were performed in Bohuslav Dvorak's lab at the University of Arizona using their rat model of experimental NEC (summarized in Figure 1). While Dr Dvorak would probably prefer that I call this a preterm NEC model, the reality is that most of the term NEC cases were of lower birth weight and trended towards a few days earlier gestation than controls in the Intermountain Health Care cohort, and Dvorak's rat pups were delivered by cesarean section just 1 day before term (so it is an appropriate model). Without going into great detail, Yoshita Ito et al. subjected their rat pups to daily asphyxia with cold stress and randomized them to either rat breast milk or formula nutrition. The latter therapy results in NEC by the end of the week, but by four days of life there were numerous changes within the microvasculature, consistent with the events preceding mucosal necrosis. These included a decrease in capillary

Rat model of Experimental NEC

Rat pups delivered by cesarean section 1 day before term<smiles>C1=CC=C1</smiles>

Daily asphyxia + cold stress $x 4$ days

$\checkmark$

Fed Rat Breast Milk<smiles>C1=CC=C1</smiles>

No change from baseline

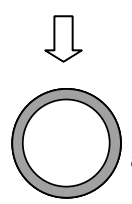

Relative arteriolar diameters after endothelin-1 application
Figure 1 Schematic summary of the publication by Ito et al. (Intestinal microcirculatory dysfunction during the development of experimental necrotizing enterocolitis, Pediatr Res; 61(2) 180-184.). A protective effect of breast milk is demonstrated upon the mucosal microvasulature, using a wide variety of assays. 


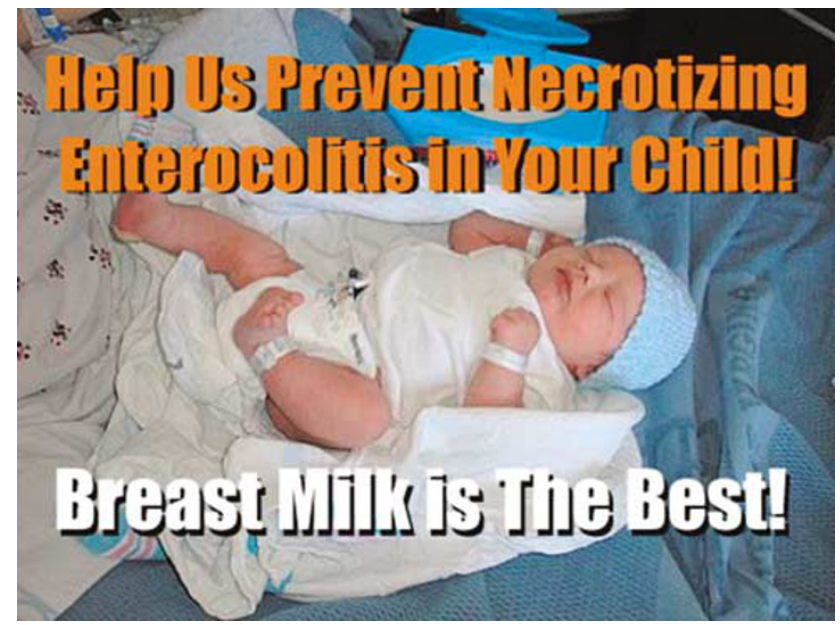

Figure 2 Suggested slogan for the front of a card to be given to mothers of term infants with preconditions associated with term NEC, with text on the back stating that 'We have identified your child as having a higher than normal risk of developing a disease known as necrotizing enterocolitis (NEC). This disease affects the intestine and can be life threatening. Up to $7 \%$ of infants with one of the risk factors your child has will get NEC if they are fed infant formula. The good news is that breast milk helps to prevent NEC. Help us prevent NEC by choosing to feed your child breast milk. Even if it is only for the first month of life, it could be the most important choice you make. Ask your doctor, nurse or lactation specialist for more details. Thank you'.

density, an increase in leukocyte adherence within venules, an increase in endothelin-1 expression, a decrease in endothelin-B receptor (one subtype of which is known to relax arterioles), and an increased arteriolar constriction in response to applied endothelin-1. Here we have a clear link between global hypoxia and stress with the microvascular perturbations that lead to NEC. Most importantly, formula permitted those changes and breast milk prevented them.

So the efficacy of breast milk has been demonstrated in rats, but what about humans? The Intermountain Health Care database shows an association between breast milk and term NEC prevention, but is there compelling evidence that breast milk actually prevents NEC? Well yes, there is in preterm infants.

Richard Schanler et al. ${ }^{6}$ published an appropriately powered, randomized trial in 2005, comparing maternal breast milk against both formula and donor breast milk in the reduction of NEC and late-onset sepsis in extremely premature infants. There was a significant decrease in NEC and late-onset sepsis in infants who got maternal breast milk when compared to the other two forms of nutrition. Now there could be many reasons why donor milk did not prove to be efficacious, including the fact that it is pasteurized, but there is little doubt that mother's breast milk is the ideal solution for NEC prevention.
This brings us back to the database that Bob Christensen has expertly engineered and the conclusions of DK Lambert et al. ${ }^{1}$ regarding term NEC. The biggest nugget in the manuscript, in this editorialist's opinion, is the capacity to identify prospectively term infants at risk for NEC. With reasonable certainty we can now project that $7 \%$ of all term infants with diagnoses of polycythemia, small for gestational age, congenital heart disease and perinatal depression will get NEC in the current climate of formula exposure. In light of the other literature we have reviewed, Lambert et al. have subtly offered us a most compelling solution for prevention of term NEC. We must target mothers of term infants with these known preconditions. We must convince them to provide breast milk to their infants at least for the first month of life at a minimum (Figure 2). This should be advocated for every neonatal intensive care unit (NICU) patient, but this population of mothers is especially likely to be successful because lactation in the term mother is inevitable (versus success being less certain in mothers of extremely premature infants). In short, this is an achievable goal and one that is well worth a targeted focus of breast milk advocacy.

Term NEC is the snow on top of the mountain when it comes to acquired neonatal intestinal diseases (preterm NEC, spontaneous intestinal perforation and viral enteritis of infancy are all more prevalent), but a targeted breast milk campaign in every NICU might just start an avalanche.

PV Gordon Division of Neonatology, University of Virginia Children's Hospital, Charlottesville, VA, USA E-mail: Pvg4n@virginia.edu

\section{References}

1 Lambert DK, Christensen RD, Henry E, Besner GE, Baer VL, Wiedmeier SE et al. Necrotizing enterocolitis in term neonates: data from a multihospital healthcare system. J Perinatol 2007 [E-pub ahead of print]; 29 March 2007; doi:10.1038/sj.jp7211738.

2 Nowicki PT, Dunaway DJ, Nankervis CA, Giannone PJ, Reber KM, Hammond SB et al. Endothelin-1 in human intestine resected for necrotizing enterocolitis. J Pediatr 2005; 146: 805-810.

3 Nankervis CA, Nowicki PT. Role of endothelin-1 in regulation of the postnatal intestinal circulation. Am J Physiol Gastrointest Liver Physiol 2000; 278: G367-G3675.

4 Nowicki PT. Effects of sustained low-flow perfusion on the response to vasoconstrictor agents in postnatal intestine. Am J Physiol 1999; 276: G1408-G1416.

5 Ito Y, Doelle SM, Clark JA, Halpern MD, Mccuskey RS, Dvorak B. Intestinal microcirculatory dysfunction during the development of experimental necrotizing enterocolitis. Ped Res 2007; 61: 180-184.

6 Schanler RJ, Lau C, Hurst NM, Smith EO. Randomized trial of donor human milk versus preterm formula as substitutes for mother's own milk in the feeding of extremely premature infants. Pediatrics 2007; 116: 400-406. 